\title{
Importancia judicial de los grupos sanguneos
}

\author{
Por el Doctor GREGORIO DURAND FLOREZ \\ Catedrático de la Universidad Católica
}

No es una novedad científica el tema de que vamos a tratar, así como tampoco lo es su aplicación legaí, pero es muy conveniente y necesario contribuir a su difusión y mejor conocimiento por parte de los estudiantes de Derecho, abogados y magistrados, a fin de que estas pruebas sean utilizadas con mayor frecuencia en el país.

Como toda innovación, estas investigaciones sufrieron un periodo de dudas antes de ser aceptadas. En Berlín hubo un famoso fallo judicial que rechazó el valor probatorio de la herencia de los grupos sanguíneos, para convertirse en la nación que, conjuntamente con Rusia, hace mayor uso de esta prueba, pero para ello se necesitó una polémica doctrinal sobre las leyes de herencia biológica, hasta que los magistrados aceptaron el valor del método, cuya aplicación es hoy universal.

\section{A).-NOCIONES BIOLOGICAS}

\section{1).-La sangre y los grupos sanguíneos.-}

Al estudiar la sangre se observan dos partes, una formada por los elementos figurados, y otra por el plasma, que es líquida. Entre los elementos figurados tenemos los glóbulos blancos, las plaquetas, y los gíóbulos rojos, que son los que en este caso, nos ofrecen mayor interés. El plasma, contiene disueltas muchas substancias, y entre ellas el fibrinógeno, que interviene en la coagulación de la sangre. El plasma y el suero son la misma cosa, salvo que el primero tiene entre sus componentes el fibrinógeno y el segundo nó. Para nosotros, en el caso de que tratamos, pueden cornsiderarse como terminos sinónimos, ya que en ambos existen disueltas las aglutininas.

\section{2).-Las aglutininas.-}

Las aglutininas de la sangre humana tienen el poder de aglutinar, bien sean los microbios y espiroquetes, bien sean ciertas células como los 
glóbulos rojos de otras especies zoológicas, o los glóbulos rojos humanos incompatibles con los del individuo estudiado.

\section{3).-Los grupos sanguíneos. -}

La terapéutica moderna empezó a usar, a fines del pasado siglo, las transfusiones de sangre humana, y ai lado de notables éxitos se registraron accidentes graves.

En 1900, Kar! Landsteime:, demuetra que existen en el suero de la sangre humana aglutininas para los globulos rojos de otrns hombres, creando así el conccpto de los grupos sanguineos, y que derominó:

$$
\text { A. D, } \quad \mathrm{B}, \quad \text { Y O (cero) }
$$

Ecta es la ciaricación més acepada, y que recomenda el Comité de Hiriene de la Liga de las Naciones. (1)

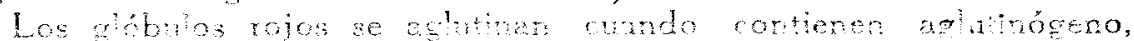
pow actón te le armininas. Esto con dos: "Ala" y "Beta" pudiendo

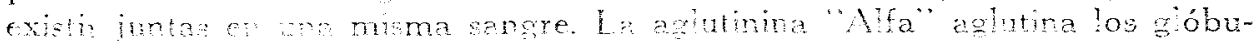
Tos roins con a Minógeno A y la "Beta" las glóbulos rojos B. Es claro. que ${ }^{2}$ sange de un perona no pueden coexistir una aplutinina con el aglutinócono compentiente. pues hatría acutinación en masa de los glóbulos rojoe. Hay cuatro combinaciones posibles:

\section{Aglutinógeno Aglutinina $\%$ en la $\%$ en el Perú}

$\begin{array}{llcrr}\text { Grupo AB (I) } & \mathrm{AB} & \text { no tiene } & 1 \% & 3,6 \% \\ \text { Grupo A (II) } & \mathrm{A} & \text { "Beta" } & 45 \% & 14,1 \% \\ \text { Grupo B (III) } & \mathrm{B} & \text { "Alfa" } & 11 & 7,2 \% \\ \text { Grupo O (IV) } & \text { no tiene "Alfa" y "Beta" } 43 \% & 75,1 \%\end{array}$

Los grupos sanguíneos están repartidos de manera diferente en la raza humana, según se observa en los porcentajes que damos, pero hay variaciones en las diferentes razas, y localidades; por ello hemos creido conveniente añadir los resultados obtenidos por el Dr. Arce Larreta en nues. tro país al estudiar a 1.372 individuos.

\section{4).-Subgrupos sanguíneos, (factores o tipos).-}

Estudios cada vez más precisos demuestran variaciones en los grupos fundamentales, formandose subgrupos, cuya importancia para aplicación judicial es considerable. Así tenemos los subgrupos:

$$
\text { A1; A2; A1B; A2B }
$$

Landsteimer, genial investigador en esta materia, ha descubierto otras propiedades que ha denominado:

\section{$\mathrm{M} ; \mathrm{N} ; \mathrm{y} \mathrm{MN}$}

de gran valor en criminología. La técnica para su demostración es muy delicada, utilizándose animales de laboratorio, y sueros "patrones", la- 
boratorios bien mortados y técnicos especializados. Landsteimer junto con Levine ha encontrado el factor $P$, más frecuente en la raza negra que en la blanca.

Solo hemos querido dar los subgrupos unánimemente aceptados por experimentación, pues creemos que son los únicos que ofrecen garan. tía a la justicia. Pero debemos añadir que hay muchos otros en estudio, y que probablemente se utilizarán en el futuro.

\section{5).-Propiedades de los grupos y subgrupos sanguíneos.}

Se admiten como hechos demostrados y comprobados:

a) El grupo sanguíneo existe en el recién nacido, y se estabiliza durante el primer año de la vida, manteniéndose invariable en eí transcurso de toda la existencia, y aún en el cadáver, hasta que los fenómenos de la putrefacción modifican la estructura química del organismo;

b) Los subgrupos siguen estas propiedades biológicas, siendo más dificil y delicada la técnica de su demostración, y desapareciendo con mayor precosidad en el cadáver.

c) El hecho de que un individuo pertenece a un grapo, se debe a la herencia, que se rige por las leyes de Mendel, propiedad demostrada por von Dugern, Hirzfeld y Bernstein, y comprobada en cientos de miles de casos.

1" Ley: "Las propiedades A y $B$ son dominantes sobre la propiedad $O$ (cero) y por lo tanto no pueden aparecer en los descendientes si no existen en los padres".

$2^{2}$ Ley: "Los padres pertenecientes al grupo 0 , no pueden tener hijos AB".

La propiedad 0 es recesiva y en algunos casos puede mantenerse oculta (híbridos). En los cruces entre A y B, las propiedades pueden no aparecer en los descendientes, dando otro caso de hibridismo. El cuadro siguiente expone las posibilidades que existen:

Padres

$O \times 0$

$A \times A$

$O \times A$

$B \times B$

$O \times B$

$A \times B$

$O \times A B$

$A \times A B$

$B \times A B$

$A B \times A B$
Hijos posibles

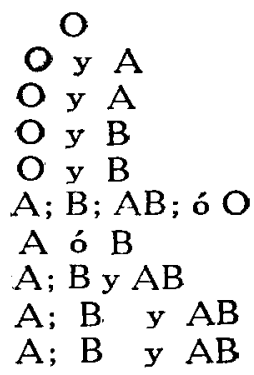

Thompsen estableció las leyes hereditarias de los subgrupos: 
3a. Ley: "A1 es dominante sobre A2 y $O$ "

4a. Ley: "A2 es dominante sobre $O$ "

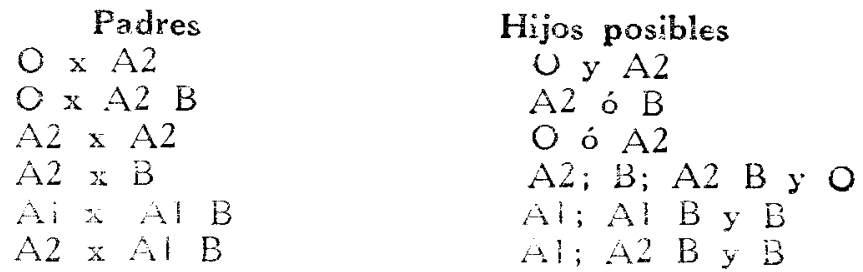

Los factores M y $\mathrm{N}$ son dominantes:

5a. Ley:"Los factoros of y fi ho puecien aperecer en la descendencia si no cxisten en los padres";

Ea. Ley: bos pridues in no preden serer hios $N, y$ los $N$ no pueden tener hijos M".

En el Feru. savo el caso citado del Dr. Aree Larreta gate ha estudiado los grupos que inclumos en el primer cuadro, no han sido investigajos los subgrupos, que acabamos de mencionar. (2)

\section{B).-DMPORTANCIA JUDREAL DE LOS GRUPOS SANGUINEOS}

El cotudo do grupos sangúneos tiene imporascia en mateme civ pax a exeusion de la fianon $y$ en ias pericias criminales en que se estudian manchas de sangre para la exchueión de sospechosos. Hacemos notar el aspecto negativo, en que no se airma, sino que se riega, y la ra zón es clara. los posibles padres que pertenezcan al stupo sanguíneo son muchos, aún en una localidad determinada: el perito no puede hacer una afirmación roincia pero si puede sega rotundamente pues es evidente que cie dos padres a ro podrá obtenerse un descendiente B.

Sin embargo es unís pucba que añadida a lo ya actiaco puede orientar a la mejor administración de justicia.

\section{6).-Aricación en los casos de Filiación.}

a) Hijos ilegitimos, cuyo presunto padre les niega el reconorimiento (negación de almentos $y$ de educación);

b) Paternidad de hijo póstumo;

c) Aduterio: hijos legítinos que el pacte ro reconoce, tada;

d) Simulación de maternicled y en los casos de matenidan dispu

e) Supiantación de menores;

f) Trueque accidental de ninos en las matemidacies; (3)

g) Abandono de menores;

h) Infanticidio;

i) Parricidio;

En Alemania y en Rusia la aplicación de estos conocimiertos es co. mún y frecuente; asi en la primera, pasan de 20.000 los casos gut han co. 
nocido los tribunales; Austria, Dinamarca, Suecia, Francia, etc., han empleado el método en menor escala.

En América, los Estados Unidos, especialmente en el estado de Nueva York, los usan cada vez más; Cuba, Chile, Uruguay y Argentina ticnen numerosos casos en su casuística forense.

7) Manchas scspechosas de sangre. Reconocimiento del origen individual.-La existencia de manchas sospechosas de sangre, en el suelo, en los muebles, en las ropas, en armas cortantes o contundentes, etc., presenta problemas muy interesantes y el auxilıo del diagnóstico del grupo a que pertenecen las manchas, permite en ciertos casos llegar a conclusiones interesantícimas para el sumario judicial. Se puede diferenciar si la sangre pertenece a la víctima o a otra persona, generalmente sospechosa. Ses puede rastrear los movimientos de ambos, siempre que pertenezcan a diferente grupo sanguíneo, pudiendo arrojar datos de importancia decisiva. (4)

Desde luego, es importante que la sangre esté fresca porque facilita grandemente la resolución del problema; con la sangre seca cólo se pue* de aplicar con seguridad la prueba de los cuatro grupos sanguíneos, siendo de menor seguridad la de los subgrupos, de allí que sea primordial que el técnico hematólogo llegue pronto al lugar del delito.

Hay otros casos, en que se ha probado que la sangre que manchaba a un arma cortante o contundente, era de diferente grupo que el de la víctima, por lo que se excluyó la posibilidad de una acción homicida o de lesiones por parte del acusado.

Debemos hacer notar un aspecto esencial de esta pericia, y es que debe confiarse a peritos muy competentes y que dispongan te laborato. rios adecuados. Los peritos existen y pueden formarse en mayor número, con el crecimiento y el auge de los Bancos de Sangre. Los laboratorios deben ser, en nuestro concepto, pertenecientes a una entidad oficial, sea el Laboratorio Central de Investigaciones de la Policía, o el Laboratorio de Policía Judicial, que debe crearse, según lo dispone el artículo 65 de nuestro Código de Procedimientos Penales. Esta es una razón más, en favor de que nuestro armamento médico - legal se perfeccione, y se aplique efectivamente en favor de la justicia.

También es importante distinguir los fracasos de los errores. Todo método tiene sus fracasos y el perito se encuentra imposibilitado de llegar a una conclusión, manifestándolo así al juez - casos de manchas viejas o modificadas por sustancias químicas-; pero ello no es patrimonio exclusivo de este método; así hay autopsias, en que no se puede llegar a conclusión valedera para el caso, porque la putrefacción ha destruído los órganos que interesaban; pero la autopsia es un método que sigue aplicándose, porque en otros casos dá indicios valiosos.

El estudio e identificación de los grupos sanguíneos tiene un valor como prueba, que el magistrado no debe subestimar, sino que al contrario debe exigir, para llegar a la más perfecta solución del caso que está juzgando. 
NOTAS. - (1) Otros autores siguen la Clasificación de Grupos I, II, III y IV, muy usada en Francia. La equivalencia es la siguiente: $A B$ -I; A-II; B-III; O-IV.

(2) Conviene indicar que hay individuos de los grupos $\mathrm{A}, \mathrm{B}, \mathrm{AB}$ y $O$ que eliminan una substancia por la saliva y Schifg ha llamado a estos. secretores o $S$, y otros que no son secretores y que denominć (s). La propiedad $S$ es dominante sobre la (s).

(3) El doctor Israel Castellanos, de Cuba, tiene un interesantísimo estudio de filiación en un caso de trueque de niños en una maternidad.

(4) El doctor Luis Sandoval Smart, del Laboratorio de Policía Técnica de la Dirección General de Investigaciones de Chile, cita un caso, entre otros muchos, que es $\tan$ interesante, que no hemos vacilado en trascribirlo:

"De los casos del año pasado, (1940), hay alguros francamente notables. A fines del año, la capital se vió conmovida por el asesinato alevoso de un distinguido industrial español, mediante golpes de martillo en la cara y cráneo".

"El estudio del rastreo hematológico hizo pensar en el primer momento, a los detectives y familiares, que el hechor pudo haber salido herido en una lucha a muerte con el occiso.

"El estudio psicológico del personal del Laboratorio, demostró que toda la sangre que se encontraba en profusión en el domitorio de la víctima, sobre las cubiertas de la cama. almohadas, almohadones. barandas de! catre, paredes, pisos y ventanas y algunos objetos que habian sobre el velador del occiso, pertenecía al mismo grupo sanguíneo, el grupo A.

"El herido, que luego falleció en la Asistencia Pública, pertenecía a este grupo y el hechor, cuyas ropas estaban manchadas con sangre de igual grupo, resultó pertenecer al grupo $A B$

"Esto, sumado a la forma de los rastros sangrientos, permitió afirmar al Juez, que no había habido lucha y que el herido lo había sido mientras dormía y que luego de haber salido el hechor, había recobrado la movilidad y, en su afán de pedir auxilio, había producido las huellas sangrientas que hicieron pensar a los iniciados en una lucha violenta.

"Las huellas sangrientas de salida, de grupo A, se debían a que el hechor llevaba las manos y la ropa ensangrentadas.

"El asesino corroboró, en su declaración, lo deducido por nosotros, y aún cuando posteriormente quiso desdecirse, las pruebas hematológicas inconmovibles, han motivado una condena a muerte, que posiblemente sea conmutada por la de presidio perpétuo, tomando en cuenta el estado mental y fisiológico del hechor". 